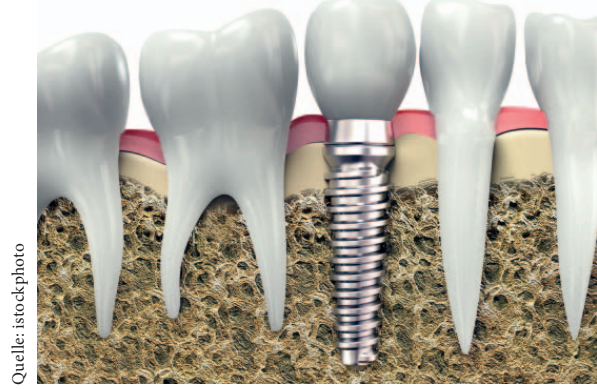

In den letzten Jahrzehnten ist die Qualität der Zahnimplantate immer besser geworden. Durch neue Materialien und neue Oberflächenbehandlungen haben sich z.B. die «Einwachseigenschaften» ständig verbessert. Immer schneller und fester werden die Gewinde in den Kiefer integriert, weil die Knochenzellen fast natürliche Bedingungen finden, um sich an dem körperfremden Material festzusetzen und es damit dauerhaft in den Knochen zu integrieren. Durch die neuen Keramikmaterialien können die künstlichen Zähne so gefärbt werden, dass sie im Kauapparat des Patienten gar nicht mehr auffallen. Von den Kosten einmal abgesehen, scheinen Implantate damit eine ideale Lösung für Patienten zu sein, bei denen eine Lücke geschlossen werden muss. In der Schweiz ist fast ein Run auf Implantate ausgebrochen, die sich binnen weniger Jahre einen festen Platz in der Zahnmedizin erobert haben. Viele Patienten fragen nach ihnen.

Doch trotz dieser Fortschritte, den Forschungsanstrengungen der MedTech-Industrie und der grossen Nachfrage durch die Patienten lässt sich bei Zahnärzten, die ganzheitlich arbeiten, ein grundsätzlicher Vorbehalt gegen Implantate nicht ausräumen. Sie gehen davon aus, dass ein im Kiefer implantierter Zahn unter Umständen für mehr Probleme sorgt, als dass er nützt.

Hinter dieser Haltung steckt die Überzeugung, dass es eine sehr enge Beziehung zwischen den Zähnen und der Gesundheit des Körpers allgemein

\title{
Zahnimplantate können problematisch sein
}

Obwohl Zahnimplantate immer häufiger eingesetzt werden, stehen ihnen viele Zahnärzte, die komplementärmedizinisch arbeiten, kritisch gegenüber. Sie glauben, dass die Implantate die Zahn-Organ-Beziehung stören.

gibt. Dabei gehen die ganzheitlichen Zahnmediziner nicht nur davon aus, dass der Zahn in den Organismus hineinwirkt, sondern es auch in der anderen Richtung eine Beziehung gibt: Ein Zahn kann ein Problem in einer anderen Region des Körpers anzeigen. Im Mundraum wird der Körper «gespiegelt», und so stehen bestimmte Teile des Gebisses für bestimmte Organe: Probleme im Zahn sind das Resultat für Erkrankungen oder Probleme in den korrespondierenden Körperregionen.

Ganzheitliche Zahnärzte gehen davon aus, dass eine Region, in der ein Zahn verloren gegangen ist, vorbelastet ist. Er sei schliesslich - ausser bei einem Unfall - nicht zufällig verloren gegangen, sondern durch eine Schwächung, die ihre Ursache ganz woanders im Körper hatte.

Deshalb müsse abgeklärt werden, ob nicht eine organische Störung im Körper vorliege, bevor man ein Implantat setzt. Anderenfalls könne das Implantat unter Umständen nicht gut genug einwachsen und drohe im Laufe der Zeit und unter Belastung verloren zu gehen. Allerdings gibt es in der Literatur keine verlässlichen Studien, die es gestatten, diesen Effekt $\mathrm{zu}$ beurteilen. Untersuchungen über den Einwachserfolg von Implantaten gehen auf die Körper-Zahn-Beziehung und deren Konsequenzen nicht ein. Zahnärzte, die einen strikt ganzheitlichen Ansatz verfolgen, lehnen es deshalb ab, Implantate zu setzen, und halten sie nur beim Zahnverlust durch einen Unfall für vertretbar.
Für viele komplementärmedizinische Praktiker ist auch die Ausstrahlung wichtig, die ein Implantat auf den Organismus hat. Einige berichten, dass sie bislang noch kein Implantat gesehen hätten, das bei einem Patienten nicht körperliche Beschwerden ausgelöst hätte. «Haben meine Probleme etwas mit meinem Implantat zu tun», sei etwa eine häufige Frage, berichtet med. dent. Sabine Vaucher. Bei den Störfeldklärungen komme immer heraus, dass das Implantat für andere Probleme in einem erheblichen Masse verantwortlich sei. Allerdings sind ganzheitlich praktizierende Zahnärzte oft auch die letzte Anlaufstelle für Menschen, die glauben, dass ihre gesundheitlichen Probleme durch ein Implantat verursacht würden.

Zahnärzte, die einen weniger strikten ganzheitlichen Ansatz verfolgen, halten Implantate im Prinzip für eine sehr gute und praktische Option. Sie lehnen es aber auch ab, die Implantate als das «Mittel der Wahl» überall dort einzusetzen, wo die Indikation stimmt und die schulmedizinischen Parameter dies zulassen. Man müsse daher vor dem Setzen eines Implantats genau abschätzen, ob es sich als Störfeld erweisen und gesundheitliche Probleme generieren könne. Entscheidend ist aber auch die individuelle Materialverträglichkeit. Diese scheint sich durch die Einführung der Zirkonoxid-Vollkeramik-Implantate verbessert zu haben. Gegenüber den Titanlegierungen sind damit für viele Patienten die Implantate besser geeignet. (rfi) 